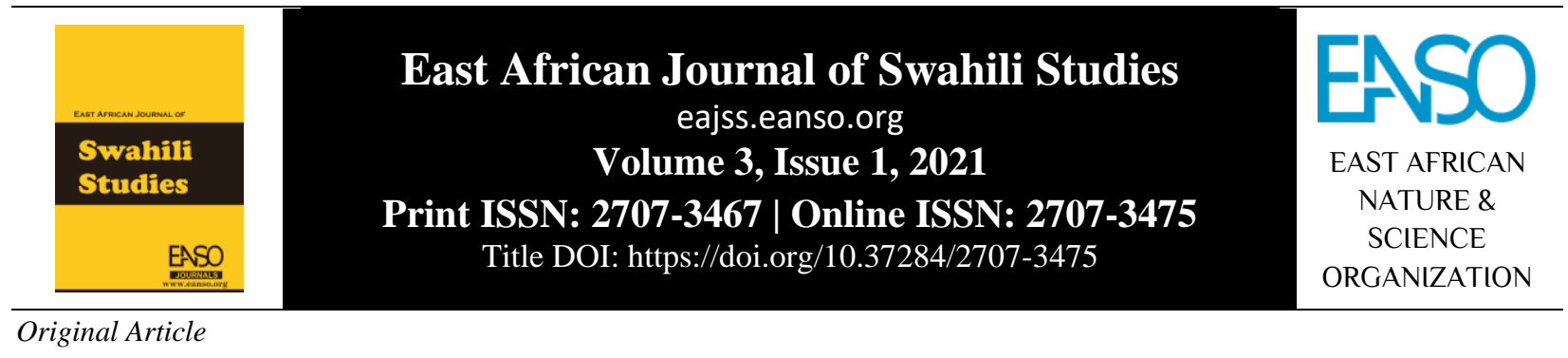

\title{
Taswira ya Mwanamke katika Kipindi cha Bi. Msafwari Runinga ya Citizen Nchini Kenya.
}

\author{
Janet Jepkurui Kibet ${ }^{1},{ }^{*}$ Dkt. Rebecca Wanjiru Omollo, PhD ${ }^{1}$ Dkt. na Rose Mavisi, PhD ${ }^{1}$ \\ ${ }^{1}$ Chuo kikuu cha Catholic, S.L.P. 4803, Eldoret, Kenya. \\ * Barua pepe ya mawasiliano: janetbett9@gmail.com.
}

DOI ya Nakala: https://doi.org/10.37284/eajss.3.1.430

Tarehe ya Uchapishaji: IKISIRI

05 Oktoba 2021 Utafiti huu unalenga kuchunguza na kutathmini taswira ya mwanamke katika

Maneno Muhimu: kipindi cha Bi. Msafwari Katika runinga ya Citizen nchini Kenya kwa mtazamo wa kifasihi. Utafiti huu uliongozwa na lengo lifuatalo: Kueleza nafasi ya mwanamke katika kipindi cha Bi. Msafwari Katika runinga ya Citizen Taswira ya nchini Kenya. Nadharia iliyotumika katika utafiti huu ni nadharia ya Mwanamke, Mwanamke, Ufeministi mathalani Ufeministi wa Kiafrika na nadharia ya mtindo. Nadharia ya Ufeministi ilifaa utafiti huu kwa kuwa inashughulika na masuala ya kijinsia hasa jinsia ya kike. Muundo wa kimfano ulitumika katika uchanganuzi wa Bi. Msafwari, Runinga ya Citizen,

Ufeministi. utafiti huu. Utafiti huu ulijikita katika maktabani ili kupata maandishi yanayohusiana na mada iliyoshughulikiwa. Kutokana na maelezo haya, kundi lengwa la utafiti ni kipindi cha Bi. Msafwari Katika runinga ya Citizen. Sampuli iliyotumika ni sampuli ya kimaksudi ambayo kipindi cha Bi. Msafwari kiliteuliwa. Utafiti huu ulitumia mbinu ya kutazama, kusikiliza na kurekodi vipindi katika kukusanya data yake. Matokeo ya utafiti yalijitokeza wazi kuwa, nafasi ya mwanamke ni: kumfurahisha mume wake, kumsaidia mume wake, kutii, kumheshimu na kunyenyekea mbele ya mume. Pia ana nafasi ya kudumisha amani katika ndoa. Ukusanyaji na uchanganuzi wa data, uwasilishaji wa matokeo ulifanywa kwa njia ya maelezo. Utafiti uliofanywa utawafaa watafiti wengine watakaojishughulisha na mada inayohusu mwanamke kwani wataweza kurejelea kazi hii ili kupata habari kuhusu nafasi ya mwanamke. Kwa ujumla, utafiti uliyofanywa ulichunguza taswira ya mwanamke katika kipindi cha Bi. Msafwari. 
East African Journal of Swahili Studies, Volume 3, Issue 1, 2021

Article DOI: https://doi.org/10.37284/eajss.3.1.430

\section{APA CITATION}

Kibet, J. J., Omollo, R. W., \& Mavisi, R. (2021). Taswira ya Mwanamke katika Kipindi cha Bi. Msafwari Runinga ya Citizen Nchini Kenya. East African Journal of Swahili Studies, 3(1), 132-148. https://doi.org/10.37284/eajss.3.1.430

\section{CHICAGO CITATION}

Kibet, Janet Jepkurui, Rebecca Wanjiku Omollo, and Rose Mavisi. 2021. "Taswira ya Mwanamke katika Kipindi cha Bi. Msafwari Runinga ya Citizen Nchini Kenya”. East African Journal of Swahili Studies 3(1), 132-148. https://doi.org/10.37284/eajss.3.1.430.

\section{HARVARD CITATION}

Kibet, J. J., Omollo, R. W. and Mavisi, R. (2021) “Taswira ya Mwanamke katika Kipindi cha Bi. Msafwari Runinga ya Citizen Nchini Kenya”, East African Journal of Swahili Studies, 3(1), pp. 132-148. doi: 10.37284/eajss.3.1.430.

\section{IEEE CITATION}

J. J. Kibet, R. W. Omollo, and R. Mavisi, "Taswira ya Mwanamke katika Kipindi cha Bi. Msafwari Runinga ya Citizen Nchini Kenya”, EAJSS, vol. 3, no. 1, pp. 132-148, Oct. 2021.

\section{MLA CITATION}

Kibet, Janet Jepkurui, Rebecca Wanjiku Omollo, and Rose Mavisi. "Taswira ya Mwanamke katika Kipindi cha Bi. Msafwari Runinga ya Citizen Nchini Kenya”. East African Journal of Swahili Studies, Vol. 3, no. 1, Oct 2021, pp. 132-148, doi:10.37284/eajss.3.1.430.

\section{UTANGULIZI}

Utafiti huu ulichunguza na kutathmini taswira ya mwanamke katika kipindi cha Bi. Msafwari katika runinga ya Citizen nchini Kenya. Duniani, suala la mwanamke ni suala ambalo limekuwepo kwa karne nyingi katika jamii na tamaduni mbalimbali ulimwenguni. Katika historia za mwanamke pale Ulaya mwanamke hakuwa na haki yeyote katika karne ya 19 na kurudi nyuma. Kwa jumla, wanawake walikuwa wake na mama nyumbani ambao hawakuruhusiwa kusoma wala kumiliki mali vilevile hawakuwa na haki yoyote ya kiuchumi. Majukumu yao hasa yalikuwa ni kuwa mama wa kutunza watoto au mke. Ikilinganishwa na karne ya sasa kuna tofauti kubwa kwa mfano inayodhihirisha kuwa kuna tofauti ni kupitia kwa ripoti ya mwaka wa 2021 kuhusu usawiri wa kijinsi, inayodai kuwa wanawake kwa wanaume, wasichana kwa wavulana wana haki ya kutodhulumiwa na kutobaguliwa na kupewa fursa ya kustawi na hata kuongoza (European Union, 2021).

Kulingana na ripoti ya Jumuiya ya Usawa wa Afrika (2015), inayoshughulikia uwezeshaji wa wanawake wa Afrika kwa ajenda na matendo imelenga kuchunguza usawa wa kijinsia baina ya mwanamke na mwanaume kwa kuzingatia masuala matatu nayo ni: Usawa wa nafasi za kiuchumi, usawa wa upataji wa haki za kibinadamu na maendeleo na usawa katika sheria na taasisi nyinginezo. Masuala haya yamesaidia katika kutambua fursa za mwanamke katika maisha yake ya kila siku.
Nchini Kenya, kwa hakika, mwanamke ndiye anayelea familia na kuiongoza. Msingi mkuu unaounda familia ni mwanamke na si mwanamme (Kawia, 2015). Inawezekana mwanaume kukosekana katika familia labda hata kutokana na maafa na kama mwanamke atakuwa na akili na umakini, anaweza kuilinda na kuiendeleza vyema familia. Lakini wakati mwanamke anapokosekana katika familia, mwanamme hawezi kuilinda na kuiendesha familia hiyo. Hivyo mwanamke ndiye anayeilinda familia (Mrikaria, 2011).

Miongoni mwa kazi muhimu zinazopaswa kutendwa ni kuwafunza wanawake mambo mazuri ya kufanya wanapokuwa ndani ya nyumba zao, yaani jinsi ya kuhusiana na waume na watoto wao. Kuna wanawake ambao ni watu wazuri wapole, wenye subira, wastahimilivu na wenye tabia nzuri, lakini hawajafunzwa njia nzuri za kuamiliana na waume na watoto wao (Mavisi, 2007). Hivyo basi, kazi hii ililenga kuhakiki taswira ya mwanamke katika kipindi cha Bi. Msafwari Katika runinga ya Citizen.

\section{SUALA LA UTAFITI}

Tafiti kuhusu mada ya taswira ya mwanamke zimeshughulikiwa na watafiti wengine. Kupitia kwa tafiti hizo tumetambua kuwa mwanamke amechorwa kwa namna mbalimbali tangu kuanzishwa kwa fasihi. Vyombo vya habari navyo vimechangia pakubwa katika kuendeleza taswira ya mwanamke. Ongezeko la vipindi katika Runinga 
mbalimbali vinavyozungumzia masuala ya wanawake ni ishara tosha kuwa vyombo vya habari vina mchango katika usawiri wa mwanamke na kutupa picha kamili ya jinsi mwanamke anachorwa katika vyombo hivyo vya habari.

Kazi nyingi za wanawake zimetalii tanzu kama riwaya, tamthilia na ushairi lakini mtafiti amekusudia kuhakiki Taswira ya mwanamke katika kipindi cha Bi. Msafwari katika runinga ya Citizen nchini Kenya. Kwa ujumla, utafiti huu ulikuwa na nia ya kuchunguza namna mwanamke amechorwa katika kipindi kiteule na kuelezea uhalisia wake katika jamii ya leo.

\section{UHAKIKI WA MAANDISHI}

\section{Nafasi ya Mwanamke katika Kipindi cha Bi. Msafwari}

Kuna watafiti wengi ambao wameweza kushughulikia suala la nafasi ya mwanamke katika mikakati mbalimbali. Kuna utafiti uliofanywa na Lantara (2015) katika Makala ya: The roles of woman as leader and housewife, anaeleza kuwa mwanamke katika maisha yake ana majukumu mazito kuliko mwanaume. Inasema kuwa nafasi ya mwanamke wa kisasa si mke tu nyumbani bali pia ana shughuli nyingi za kikazi. Inaeleza kuwa nchi ya Indonesia nafasi ya mwanamke ni: kuwa mke anayemuunga mkono mume wake kwa kila hali kama mke mpendwa na rafiki wake wa karibu ili wajenge familia iliyo na furaha. Kutokana na Makala haya tulitambua kuwa kazi hii itatufaa kwa vile ilishughulikia masuala ya nafasi ya mwanamke.

Miongoni mwa watafiti wengine ni Sawe na wenzake (2016) katika kazi yao ya Taswira ya mwanamke katika ndoa mintarafu ya utendi wa mwana kupona. Kulingana na wao mwanamke amechorwa kama mtunzaji wa mume wake yaani anafaa kumtunza mume wake kwa kila hali. Mwanamke amepewa wosia wa kumtunza mume, hivi kwamba mume anapolala, mwanamke asiwe mbali naye bali amkaribie ili akihisi joto aweze kumpepea vilevile kumpapasa asihisi upweke. Kando na hayo mwanamke amechorwa kama mfanya kazi katika nyumbani. Wanadai kuwa ni jukumu la mwanamke kumpikia mume, kuosha nguo za mume na watoto wake na ni kazi ya mwanamke kuhakikisha kuwa mume ameshughulikiwa vilivyo. Mbali na hayo mwanamke anapewa jukumu la kudumisha amani katika ndoa. Kwani anashauriwa achunge na autumie ulimi wake vizuri ndiposa ndoa yake idumu. Kasuma (2014) katika kazi yake anadhihirisha kuwa nafasi ya mwanamke ni kuwa mtetezi wa wanyonge. Anadai kuwa wahusika wakuu ni wanawake vijana hii ni ishara ya mabadiliko ya kizazi, hali inayobainisha nafasi ya kizazi cha vijana katika kutetea usawa wa kijinsia na vilevile kumkomboa mwanamke kutoka pingu za kitamaduni ambazo zimemfungia nyumbani. Kulingana naye ujana wa wahusika umetumika kuashiria kutimika kwa enzi za dhuluma na kutimilika kwa wakati wa mabadiliko katika jamii. Hivyo, kazi hii ilitufaa katika kazi yetu kwa vile, alimchukua mwanamke kama mtetezi wa wanyonge vilevile katika kazi yetu mwanamke anajitetea.

Saro (2017) katika tasnifu yake anasema, mwanamke ni kiumbe ambaye anatazamwa kuwa mtiifu wa hali ya juu kwa wazazi, walimu na kwa mume baada ya kuolewa. Mtazamo huu unatokana na maumbile ya mwanamke kuwa ni kiumbe mtulivu, mwenye huruma na asiyekuwa na makuu. Kazi yake ilitufaa kwa sababu inachunguza usawiri wa mwanamke na inatofautiana na kazi yetu kwa sababu inaangazia Riwaya na yetu inaangazia vyombo vya habari.

Sawe (2018) anashughulikia usawiri wa mwanamke katika ndoa. Anasema kuwa, mwanamke na mwanaume wanafaa kuheshimiana, kufurahiana na kutumia lugha nzuri, kuwepo kwa mahaba, kuthaminiana, kuvumiliana, kuwepo kwa unyenyekevu, kupendana na kushikilia imani za dini. Sehemu hii imetuwezesha kuchunguza na kubainisha uhalisia wake katika kipindi kiteule. Kazi hii itatusaidia kwa sababu inashughulikia usawiri wa mwanamke hivyo, kutuelekeza namna mwanamke anachorwa. Kazi yake ni tofauti na yetu kwa sababu, anaonelea kuwa mzigo anaotwikwa ni jukumu lake, lakini kulingana nasi kuna baadhi ya mambo hayafai kuhesabika kama jukumu bali ni unyanyasaji.

\section{MISINGI YA NADHARIA}

Nadharia zilizotumika katika utafiti huu ni Nadharia ya Ufeministi mathalan Ufeministi wa Kiafrika na Nadharia ya Mtindo. 


\section{Nadharia ya Ufeministi}

Murungi (2013) anadokeza kuwa, neno 'Ufeministi' linatokana na neno la Kilatini 'Femina' linalomaanisha mwanamke. Dhana hii inamanisha uwakilishaji wa haki za wanawake kufuatana na imani ya usawiri wa kijinsia. Ufeministi ni Nadharia inayojikita na masuala ya wanawake na inajaribu kufichua matatizo wanayoyapitia wanawake. Ufeministi hujumlisha aina nyingi za misimamo, itikadi na matapo tofauti tofauti ambayo hutetea haki za wanawake dhidi ya ubaguzi wa jinsia uliotawala dunia kwa muda katika masuala ya siasa, uchumi, utamaduni, dini na jamii kwa ujumla. Irib (2014) anaeleza kuwa, Ufeministi ni harakati ya ukombozi zilizoanzishwa na wanawake ili kupinga mfumo dume uliowakandamiza wanawake katika nyanja mbalimbali.

Masuala ya kifeministi yalianza kuzungumziwa katika karne ya kumi na tisa ambapo yalishika kasi miaka ya sitini na sabini ya karne ya ishirini. Kwa hivyo, Nadharia ya Ufeministi inajulikana kama iliibuka katika muungano wa ukombozi wa wanawake uliopamba moto katika miaka ya sitini pale Marekani (Ntaragwi, 2004).

Ramton (2015) Nadharia ya Ufeministi ulianzishwa kule Ugriki wa kale na waasisi wake wakiwa Sappoh, Hildegard wa Bingen, Christine de Pisan, Olmpes de Gouge, Mary Wollstonecraft na Jane Austen wote walipigania heshima na haki ya msingi wa kibinadamu ya kijinsia ya kike. Wasomi na Wanahistoria hugawanya harakati za wanawake katika mawimbi matatu makuu. Wimbi la kwanza la Ufeministi lilitokea mwishoni mwa karne ya kumi na tisa na mwanzoni mwa karne ya ishirini, iliyotokea mjini katika mazingira ya viwanda na siasa za kijamii. Lengo la wimbi hili lilikuwa, kupigania haki ya wanawake katika kupewa fursa ya kupiga kura. Wimbi la pili lilishika kasi mwanzoni mwa mwaka wa 1960 na likaendelea hadi miaka ya 1990. Wimbi hili liliibuka baada ya vita vya kidunia vya pili, wakati ambapo wanawake wengi waliingia kazini. Wimbi hili lilinuia kupitisha usawa wa haki katika urekebishaji wa katiba na kuhakikisha kuwa kuna usawa wa kijinsia. Lilisisitiza kuwa wanawake wanafaa kuwa na haki sawa za kijamii, kisiasa, kisheria na kiuchumi sawa na mwanaume. Wimbi la tatu la Ufeministi liliibuka katikati ya miaka ya 90. Moja wa muasisi wa wimbi la tatu ni Rebecca Walker, mwanamke aliye na umri wa miaka 23, aliyeunda neno, 'Ufeministi wa wimbi la tatu'. Kulingana naye wimbi la pili kihistoria lilishindwa kuingiza sauti za wanawake wengi wachanga, wanawake wasio na jinsia moja na wanawake wa rangi (Rampton, 2015).

\section{Ufeministi wa Kiafrika}

Nadharia ya Ufeministi wa Kiafrika iliibuka mwanzoni mwa karne ya ishirini na iliasisiwa kwa mtazamo wa mwanamke wa Kiafrika. Waasisi wa Nadharia hii walikuwa na mitazamo sawa juu ya masuala ya mwanamke wa Kiafrika, na waasisi wake ni pamoja na Filomena Steady (1981).

Adeola (1990) anasema kuwa, ingawa Ufeministi wa Kiafrika ulishughulikia masuala ya Kiafrika, uliendelezwa na mataifa ya Kimagharibi kupitia kwa wanawake wa rangi nyeusi na wenye asili ya Kiafrika. Katika jamii nyingi za Kiafrika mwanamke amesawiriwa kama kiumbe duni na ametegemewa nafasi inayoonekana kuwa chini lakini iliyo na majukumu mengi. Anasisitiza kwa kusema kuwa, mwanamke anatukuzwa kwa kupewa majukumu mazito huku akiendelea kubaguliwa na kutengwa kwa maamuzi ya kijamii na hata kunyanyaswa.

Steady (1981) anadokeza kuwa, Ufeministi wa Kiafrika umejikita katika mwelekeo wa kuangalia majukumu tofauti ya kijinsia kama yanayoegemeana na kukamilishana. Anasema kuwa, majukumu haya yanaenda sambamba na jinsia na hata kusababisha hali ya usawa. Masuala ya ubaguzi, uana, utabaka na mielekeo ya utamaduni huletwa pamoja ili kuanzisha Ufeministi unaomwangalia mwanamke kama kiumbe na si kwa msingi wa kijinsia. Hivyo nadharia hii itafaa sana katika uchambuzi wa data zinazohusu ujinsia katika kukamilisha lengo la utafiti huu.

Katika kushughulikia nadharia yeyote ile inafaa idhihirike kwamba kuna baadhi ya mihimili ambayo ni ya kimsingi. Kwa hivyo, katika Nadharia ya Ufeministi tutatumia mihimili ifuatayo:

Kwanza usawa wa kijinsia ambapo, Nadharia hii ina nia ya kuleta usawa wa kijinsia katika jamii. Ntaragwi (2004) anasema, baadhi ya mambo yanayozungumziwa na muungano wa ufeministi ni kuunda upya uhalisia kwa kupanga kudhihirisha 
miundo za kijadi ambayo inamtukuza mwanaume na kumdhalilisha mwanamke. Hivyo, inapigania jamii mpya inayothamini na kuheshimu jinsia ya kike. Hii ilitumika katika utafiti kuonyesha uhusiano kati ya mwanamke na mwanaume tulichambua kuhusu mchango wa mwanamke katika ndoa.

Pili usanii wa kuzingatia vipengele vya fasihi. Wafula na Njogu (2007) wanaeleza kuwa, nadharia ya kifeministi inaongozwa na misingi ya kutumia fasihi kama jukwaa la kueleza kwa uyakinifu hali ya mwanamke. Kwa hivyo, Nadharia hii inatumia fasihi kama jukwaa la kuelezea hali ya mwanamke katika jamii. Jazanda kama fani mojawapo ya fasihi ni mwafaka katika kuchunguza na kueleza jinsi mwanamke anachorwa katika jamii. Kazi hii ilikuwa inatumia tamathali ya usemi ya jazanda katika kumsawiri mwanamke.

Tatu nadharia hii inalenga kutambua kuwa wanawake wote sio dhaifu kama inavyodhaniwa bali pia kuna wanaume ambao ni dhaifu kuliko wanawake. Kuna haja ya wanawake kuungana ili waweze kushinda mfumo wa ubabe na kuleta usawa wa kijinsia. Mihimili hii ilitusaidia katika kubainisha ukweli huo kuwa kuna wanawake ambao sio dhaifu kutoka kwenye mazungumzo katika kipindi cha Bi. Msafwari.

Mwisho, hadhi ya mwanamke. Utafiti huu umeshughulikiwa kwa msingi wa kukisiwa kuwa mwanamke ana hadhi ya chini katika jamii. Hivyo pana haja ya kubuniwa kwa sheria ili mwanamke aweze kutumia hiyo hadhi inayoonwa kuwa duni kusimama na kupigania hadhi yake. Nadharia hii ilitufaa kwa vile inazingatia utamaduni na tamaduni yenyewe inamkandamiza na kumdunisha mwanamke hivyo kazi hii ilisaidia katika kuonyesha hali halisi ilivyo na hii ilidhihirika wakati tulipokuwa tunashughulikia na nafasi ya mwanamke katika kipindi kiteule. Utafiti huu ulihakiki vipindi vya Bi. Msafwari katika runinga ya Citizen, ili kuonyesha jinsi kinavyomsawiri mwanamke.

\section{Nadharia ya Mtindo}

Nadharia hii iliasisiwa na Buffon (1930). Buffon anadai kuwa mtindo ni mtu mwenyewe. Kwa kuwa na maana kuwa ni ule upekee wa mtu katika matumizi yake ya lugha ili kuwasilisha ujumbe fulani.

Miongoni mwa waasisi muhimu wa nadharia hii ni Coombes (1953:10) akinukuliwa na Oiko (2017) anasema,

Mhakiki ni lazima azingatie vipengele vinavyofanya kazi fulani iwe ya kuvutia. Nia ya mtindo ni kuchunguza na kuchanganua lugha na kaida zake kwa nia ya kutambua mbinu zilizotengwa kwa kazi fulani ya kijamii, kufafanua sababu ya vipengele fulani kutumiwa na vingine kuachwa.

Kulingana na maelezo haya, ni dhahiri kuwa nadharia ya mtindo huangazia namna msanii anavyotumia lugha katika kuwasilisha ujumbe wake. Miongoni mwa wataalamu wanaunga mkono madai hayo ni pamoja na (Mosha, 2002; Wamitila, 2013). Wanakubaliana na madai haya kuwa, maana ya kazi ya fasihi hujikita katika mtindo aliotumia mwandishi katika matini husika.

Nadharia ya mtindo, huhusisha maelezo ya kiisimu ili kuweza kufafanua dhana zake za kimsingi katika fasihi. Leech (1963) anaeleza kuhusu mahusiano uliopo baina ya fasihi na isimu na yanadhihirisha kuwa dhana hizi zinakamilishana katika utendaji kazi. Anasema kuwa:

Mtindo hauhusiani tu na ufasiri wa maana za matini kwa mtazamo wa kiisimu. Nadharia hii kama taaluma haijisimamii pweke bali

huhusisha vipengele vya kifasihi na vipengele vya kiisimu katika uhakiki. Hii ni kwa sababu ya ugumu uliopo katika kuufafanua msamiati mpana wa kimsingi wa kihakiki katika fasihi kama vile, taswira, sitiari, kinaya, jazanda na usambamba bila kurejelea dhana za kiisimu. Kwa hivyo, katika kuziunganisha taaluma hizi mbili ya fasihi na isimu, nadharia ya mtindo inakuwa jukwaa la kushughulikia dhana ya fasihi na isimu kwa pamoja."

Kwa ujumla, Leech anaashiria kuwa, uhakiki wa mtindo huhusu, kuchanganua vipengele vya fasihi vile vile vya isimu. Hakuna kipengele moja wapo ambacho huweza kujisimamia pweke na kujifafanua kikamilifu kati ya fasihi na isimu. Hii inaonyesha kuwa, mtindo hutumia maelezo ya isimu na fasihi katika kufafanua kazi mbalimbali. 
Kwa hivyo, tunapohakiki mtindo katika mkabala wa fasihi hatuwezi kupuuza matumizi ya vipengele vya kiisimu.

Leech, anafafanua kuwa, lengo la nadharia ya mtindo ni kuchunguza lugha na kanuni zake ili kubainisha mbinu ambazo zimetengewa kazi fulani ya jamii. Anasisitiza kuwa nadharia ya mtindo hulenga kufanikisha uchunguzi wa kina wa matumizi ya lugha katika fasihi. Naye Simpson (2004) anasema kuwa, nadharia ya mtindo husisitiza kuwa, kila kazi ya fasihi ina ubunifu ambao unapochanganuliwa kwa undani husaidia uelewaji wa undani wa matini za fasihi.

Kulingana na Wamitila (2003), mitindo ni dhana inayotumiwa kurejelea taaluma inayohusu uchunguzi na uchanganuzi wa namna mbalimbali za kuelezea matukio katika kazi ya fasihi. Anasema kuwa, ni taaluma iliyo kati ya uchunguzi wa lugha na uchunguzi wa fasihi.

Mihimili iliyotumiwa katika nadharia hii ni: kwanza ni kuonyesha kuwa mtindo wa kazi ya fasihi ndio hutumiwa na msanii kuwasilisha ujumbe wake. Mihimili hii ilitumiwa katika kufafanulia mitindo yanayojitokeza katika kipindi kiteule. Hivyo nadharia hii ilitusaidia katika kuonyesha mbinu za lugha zilizojitokeza katika mazungumzo kwenye kipindi kiteule.

Pili kuonyesha kuwa, kuna uwiano kati ya ujumbe na lugha katika kazi ya fasihi na vipengele hivi viwili haviwezi kutenganishwa. Nadharia hii ilitusaidia katika kuwasilisha kazi yetu kwa njia ya maandishi. Kwa vile ilionyesha kuwa, lugha ni chombo kinachosaidia kuelewa ujumbe uliodhamiriwa na mzungumzaji.

\section{MUUNDO WA UTAFITI}

Muundo wa utafiti ni umbo, mpango au mwongozo utakaoelekeza mtafiti kukusanya, kuchanganua na kuwasilisha data (Wanjala, 2020). Kwa hivyo, muundo uliotumika katika utafiti ni wa kimfano, mathalani mkabala wa kifasili, kwa kuwa data iliyokusanywa na kuchambuliwa ni ya kithamano. Muundo wa kimfano ni mbinu nzuri inayomwelekeza mtafiti apate data anazohitaji katika ukusanyaji wa data (Creswell, 2009).
Mkabala wa kithamano ulitumika katika ukusanyaji wa data. Kithamano ni njia ya kukusanya na kuchambua data kwa mfumo wa maelezo pasipo kufuata utaratibu wa kitakwimu (Kothari, 2004). Data hiyo itamwezesha mtafiti kufafanua taswira ya mwanamke katika kipindi cha Bi. Msafwari nchini Kenya.

Kutokana na maelezo hayo basi, utafiti huu ulitumia mkabala wa Kithamano katika ukusanyaji wa tanzu zilizotolewa katika kumsawiri mwanamke katika kipindi cha Bi. Msafwari. Hii ni kwa sababu, uwasilishaji wa tanzu hizi ulijikita katika usemaji ulioambatana na uwasilishaji wa tanzu hizi.

\section{Mahala pa Utafiti}

Utafiti huu ulijikita mtandaoni na kwenye maktaba. Vipindi mbalimbali vya Bi. Msafwari vilitazamwa kutoka kwenye mtandao wa YouTube ambapo mtafiti alisaidika katika kupata data kuhusu usawiri wa mwanamke kwenye vipindi hivyo. Aidha, mtafiti alivitazama vipindi vya kipindi cha Bi. Msafwari kutoka kwenye mtandao wa YouTube ambapo baadhi ya vipindi hivyo vilichaguliwa ili vihakikiwe.

\section{Ukusanyaji Data}

Kombo na Tromp (2006) wanasema, mbinu ambazo hutumika kukusanya data huathiriwa na vifaa vya kukusanya data. Mtafiti alitumia mbinu za kutazama na kusikiliza vipindi, na kurekodi vipindi. Mwanzo mtafiti alikitazama na kusikiliza kipindi kiteule. Mbinu ya kusikiliza ni mbinu ya kukusanya data isiyoshirikishi kwa kuwa mtafiti hatagusani moja kwa moja na watafitiwa bali hupata habari kupitia njia nyingine (Mugenda na Mugenda, 2003). Kwa hivyo uchunguzi usio shirikishi ulitumika kutazama na kusikiliza vipindi vya Bi. Msafwari kutoka kwenye runinga ya Citizen na kwenye mtandao wa YouTube.

\section{Uchanganuzi na Uwasilishaji wa Data}

Data iliyopatikana ilichanganuliwa kulingana na malengo ya utafiti. Masuala yaliyojitokeza yanayohusiana na taswira ya mwanamke katika kipindi cha $\mathrm{Bi}$. Msafwari yalichanganuliwa. Uchanganuzi sahili ulitumika wakati wa kuchanganua data iliyokusanywa kutokana na kutazama na kusikiliza vipindi vya Kipindi cha Bi. 
Msafwari. Mtafiti alipanga matokeo ya utafiti wake kwa kuzingatia maswali ya utafiti. Matokeo hayo yaliwekwa wazi kupitia kwa njia ya maelezo ili kuonyesha wazi masuala yote yaliyochanganuliwa.

Data ilichanganuliwa kwa mkabala wa kithamano ambapo mtafiti alichanganua matokeo ya utafiti. Aidha, mtafiti alichanganua taswira ya mwanamke kutoka kwenye kipindi kiteule kwa kuzingatia malengo ya utafiti.

Baada ya ukusanyaji na uchanganuzi wa data, uwasilishaji wa matokeo ulifanywa kwa njia ya maandishi na maelezo. Uwasilishaji huu ulihusu maelezo ya vipengele ambavyo vilijitokeza katika kipindi cha Bi. Msafwari katika runinga ya Citizen.

\section{MATOKEO}

\section{Mwanamke Amechorwa kama Chombo cha Kumfurahisha Mwanaume}

Asilimia themanini na saba nukta tano $(\mathrm{N}=21)$ ya vipindi viliyoteuliwa vimemchora mwanamke kama chombo cha kumfurahisha mwanaume. Baadhi ya vipindi vinashikilia kwamba, mume anapotoka kazini na amechoka na hana raha ni jukumu la mwanamke kuleta furaha na kumfanya mumewe kuwa mchangamfu. Hii inadhihirishwa katika kipindi cha "kwa nini mwanaume haridhiki na mke mmoja", Bi. Msafwari anawaelekeza wanawake watabasamu kila wakati wanapowaona waume zao. Anasema:

... ikiwa uko nyumbani ukiwa unaona mume tabasamu. Unatakikana kama mwanamke wewe ukue Rose katika yule mume, ye akitoka kazi hata kama mmetoka pamoja, lakini akukute wewe una uchangamfu fulani."

Kulingana naye kazi ya mke ni kumfurahisha na kuwa kivutio kwa mume wake, amemwambia mwanamke awe 'Rose'. Rose ni ua la waridi. Waridi ni ua nzuri na la kuvutia hivyo watu wengi huvutiwa na uzuri wake pamoja na harufu yake nzuri. Mke anapoambiwa awe Rose kwa yule mume ni jazanda tu kwa vile haimanishi awe ua lakini anashauriwa alete ule uzuri alionao katika nyumba yake ili mumewe aweze kufurahi na kuvutiwa naye kama vile ua la waridi huwavutia watu. Kwa hivyo hii ni ishara kuwa nafasi ya mke ni kumfurahisha mume.
Kipindi cha uzuri wa nyumba ni mlango, Mwanamke anaendelea kuchorwa kama chombo cha kumburudisha na kumfurahisha mume wake pale Bi. Msafwari anaposema kuwa,

“... na mwanamke hajitengenezi kwa sababu ya yeye mwenyewe, anajitengeneza kwa sababu ya mume wake, kujipamba kwako si kwa sababu ya wewe mwanamke, kujipamba kwako ni kwa sababu ya mume wako."

Hii ni ishara tosha kuwa kulingana naye dhima ya mwanamke ni kumfurahisha mwanaume bali si kujifurahisha yeye mwenyewe.

Nacho kipindi cha Majukumu ya mwanamke kimegusia suala hili la mwanamke kumfurahisha mwanaume. Kuna pale ambapo Bi. Msafwari anawashauri wanawake watumie mahaba ili kuwafurahisha na kuwaridhisha waume zao. Anasema,

“... sikiza mwanangu, lazima tujifunze mahaba. Ikiwa hutatumia 'malove,' mapenzi ni kama mgomba ama ua unapopanda mgomba au ua lako nyumbani, si huwa unalitia maji, si walipalilia, walitia hata na mbolea?

Maneno haya yanaelekezwa kwa mwanamke kuwa ni sharti afanye kila juhudi kuhakikisha kuwa mapenzi yao yanabaki kama mwanzo, na anaweza kudumisha mapenzi hayo kwa kuhakikisha kuwa anamfurahisha mume wake

Aidha, kipindi cha Athari za pesa kwenye ndoa ambapo mkala anasema:

"umetaja paka, sasa yule paka mkunekune kichwa atasikia raha... kwa hivyo mkunekune paka wako usipomkuna kuna mtu atamkuna."

Kutokana na usemi huu paka ndiye mume ambaye anastahili kukunwa kichwa. Hii inaonyesha kuwa ni sharti mke ajitahidi kadri anavyoweza kumfurahisha mume wake. Asipojitahidi atapokonywa na wanawake wengine.

Katika kipindi cha Maswali na mawaidha ya Bi. Msafwari kuna kauli anayotoa Bi. Msafwari kwamba:

"Huu usmart ambao wasema wanawake wawe wasmart, mwanamke ni ua la tena ... tafuta nguo 
nzuri, usiseme ile ni ya Krismasi mvalie tu akikuona mwenyewe ashtuke."

Kauli hii inamanisha kuwa kama mwanamke, unafaa kuwa nadhifu na ujirembeshe ili umvutie mwanaume. Mwanamke anafaa kung'ara, kupendeza na kuvutia hivi kwamba, mwanaume akiwa naye anavutiwa na kufurahia kukaa naye.

Kipindi kingine ambacho kinaunga mkono suala hili la mwanamke kumfurahisha mwanaume ni kipindi cha Mke kumtunza mume ambapo kuna kauli iliyonukuliwa inayosomwa na Rashid kwamba:

"Once he takes off his bag, literally go and wash him, scrab him, dont let him do anything himself. Do it clean his ears, clean like how you could wash a baby, do that. Dry him put mafuta on his body, dress him."

Kupitia kwa kauli hii ni wazi kwamba, nafasi ya mwanamke ni kumfurahisha mwanaume. Anapewa majukumu hayo yote kwa ajili ya kumfurahisha mwanaume. Kutokana na kauli hii ina maana kuwa mwanamke anahimizwa kujaribu kwa kila juhudi kumfurahisha mwaume hata kama ni kujitolea kifedha.

Pia nafasi ya mwanamke kumfurahisha mwanaume imejitokeza katika kipindi cha, Mbinu za kuboresha ndoa ambapo Rashid anasoma ujumbe wa mtazamaji anayesema:

“...mke anaweza kupika chakula kitamu hata kama sio kila siku hata mara moja kwa wiki. Halafu kawashawasha mishumaa pale nyumbani ili bwana akija kidogo kuna amani."

Ujumbe huu unaonyesha kuwa nafasi ya mwanamke ni kuhakikisha kuwa mume anafurahia mazingira yake. Msikilizaji huyo anasema kuwa, mke awashe mishumaa kuwasha mishumaa ni kujipamba na kuhakikisha kuwa kila kitu kiko sawa nyumbani kwa minajili ya mwanaume.

Waaidha, katika kipindi cha, Maswali kuhusu masuala ya ndoa, Mkala anasema,

“... kuna mwanamke unaweza kupita kwake nyumbani, yaani amevaa nguo imepasuka. Lakini mwambie kuna harusi anavaa nguo nzuri ya kupendeza. Sasa unashindwa huyu ni kwa sababu anaenda sokoni kule anatafuta mume mwingine maanake mimi hapendezi kwangu."

Kuna mahali ametaja kuenda sokoni, sokoni ni neno la kijazanda kwa vile, haimanishi kuwa huyo mke anakwenda sokoni mahali pa kuuza bidhaa mbalimbali, anamaanisha kuwa anakwenda kujionyesha na kutafuta mume mwingine anapovaa vizuri. Usemi huu unaonyesha kuwa kila mwanamke anapovaa vizuri, anavaa ili kumfurahisha mwanaume na si kwa sababu ya kujifurahisha na kuridhisha nafsi yake.

Katika kipindi cha, Katika ndoa ni kitu gani huchukiza wanaume, Bi. Mariam anasema kuwa:

“...kabla mwanaume hajamuingiza mwanamke ndani, wanaanza suala la uchumba. Sisi wanawake mara nyingi tunakuwa na kampeni sana. Yaani kampeni ya kumshawishi mwanaume na mwanaume akashawishika..."

Hapa kampeni haina maana ya kampeni za kisiasa bali ni kampeni ya kujipendezesha ili uvutie mwanaume. Hivyo kulingana naye anaona kuwa ili mwanamke aweze kupata mchumba ni sharti uhakikishe kuwa unafanya kila uwezavyo kuhakikisha kuwa unamvutia mwanaume usipofanya hivyo, huenda usipate fursa ya kuolewa.

Katika kipindi cha Zipi sifa za Husband material, Bi. Miriam anasema:

"Mwanaume anaweza kukuambia, una miguu mizuri lakini haitoshi, anakuambia una sura nzuri, kumbe anakudanganya ili akupate. Akishakupata hana haja na wewe tena."

Kutokana na kauli hii maumbile ya mwanamke yanatumika katika kumsifia na katika kumshawishi katika kushiriki mapenzi. Ambapo mwanamke anatazamwa kama mtu wa kumburudisha mwanaume hivyo kushawishiwa akishakubali kushiriki katika mapenzi anaachwa na mwanaume hivyo, kuwa kama chombo cha kujiburudishia.

Jambo la mavazi yanayoweza kumvutia mwanamume laangaziwa kwenye kipindi cha mjakazi kumkosoa mke mwenye nyumba mwanamke anaruhusiwa kuvaa nguo fupi na anapokaa mbele ya mume anapaswa kukaa kwa njia itakayomvutia kutaka kuwa naye. Mwanamke 
anapaswa kumvutia mume wake wakati wowote kwani mwanamume anaweza kuendeleza mahaba mahali popote kwa mfano jikoni anaweza zima gesi na kuendeleza.

Isitoshe Mkala anaongezea kuhusu mwanamke kumfurahisha mwanamume katika kipindi cha vigezo vya wanaume kuhusu ndoa anasema kuwa, wataalamu wa mapenzi husema kwa mwanamke kuwa, alfajiri mchukue mume wako kama mwanao, mchana mchukulie bwanako kama kakako na jioni mchukulie bwanako kama hawarake. Kwa hivyo hii ni kazi ya siku nzima ya kumfurahisha mwanamume.

Katika kipindi cha mwanamke mpumbavu na mwanamke mwenye hekima $\mathrm{Bi}$. Msafwari anasema kuwa mwanamke mwenye hekima huamka mapema na kumtengenezea mumewe kiamsha kinywa. Mwanamke huyu yuapatikana katika Zaburi 31 naye mume wake anamwita mke wangu wa heri. Anaongeza kuwa wanawake wa heri walibaki wachache. Isitoshe mwanamke atenge wakati wa kumfulia mume wake hivyo kumfurahisha.

Kipindi cha, Ni kosa kujuana na mtu mtandaoni ilhali soko zote zimeingia mtandaoni? Rashid anasema:

“...ni ukweli Mwenyezi Mungu amejua kuwaumba wote mabinti ukiwaangalia mtandaoni wamejitahidi warembo wako kwa sura na maumbile"

Hii inaonyesha kuwa wanawake wengi walioko kwenye mtandao wamejirembesha kwelikweli na wanajirembesha ili kuwafurahisha watu hasa wanaume. Hata katika kipindi cha Dalili gani zakuonyesha mwanandoa yuko hatarini tunatambua kwamba mwanamke amepewa jukumu ambalo si la kumfurahisha mume pekee bali hata kufurahisha wanajamii. Kwani, Lulu anasema:

"Pale unapoenda nyumbani mama anakuambia urudi, ili ujisitiri na usitiri familia."

Hii ni dhahiri kuwa pia jamii imechangia katika hali hii ya kumfanya mwanamke kumfurahisha mwanaume. Kwa vile unapotoka kwako kwa sababu zako mwenyewe jamii haiwezi kukuelewa na itakuona kama umeshindwa na nyumba. Hivyo, kufanya hata jamaa zako kukulazimisha urudi kwako kwa sababu ya kuogopa aibu.

Aidha suala hili pia linajitokeza kupitia kipindi cha nini maana ya usafi kwa wanandoa ambapo, Bi. Msafwari anasema:

“...nilikuwa katika road show nikaenda kwa mama fulani. Nilipofika nikakuta nguo zimejaa kitandani nikashangaa kitanda hiki ndicho kinalaliwa na mume"

Hivyo kutokana na usemi huu ni dhahiri kuwa sio mwanamke ajitengeneze tu kumfurahisha mume bali atengeneze nyumba pia kwa ajili ya kumfurahisha mwanaume. Mume anafaa kupendezwa na kila kitu anachotengeneza mwanamke.

Masuala hayo yote yanaonyesha kuwa mwanamke anafaa kumfurahisha mwanaume. Pia tulitambua kuwa sio wanawake pekee wanaowafurahisha watu wengine bali kuna baadhi ya vipindi ambavyo vilionyesha kuwa mwanaume pia ana jukumu la kumfurahisha mwanamke kwa mfano katika kipindi cha, mbinu za kuboresha ndoa, Mkala anasema:

"Nafikiri kosa linatokea kwetu sisi wanaume. Ni kwamba, sisi ni wawindaji bwana, kwa vyovyote awe mke wako kwa hivyo utafanya kila njia ili umpate mwanamke."

Anaposema wao ni wawindaji, anamaanisha kuwa kama wawindaji wanavyojihami na silaha ya kutumia wakiwa mawindoni vivyo, hivyo mwanaume anafaa kujitayarisha anapokuwa akitafuta mchumba. Ahakikishe kuwa anamfurahisha huyo mwanamke kwa kutumia njia yoyote ile hata kama ni kutumia uwongo.

Pia mwanaume anadhihirishwa kama kiumbe cha kumfurahisha mwanamke kupitia katika kipindi cha, Zipi sifa za Husband Material pale Rashid anasema:

“...sifa zinakwenda tofauti na ni maswala ambayo yanafanyika. Ambapo, unaona watu kila siku wanakwenda gym sababu wanaimani kwamba, mtu akishatengeneza mwili atabahatika."

Katika usemi huu ni dhahiri kuwa mwanaume pia hujitengeneza kwa sababu ya mwanamke. Yeye

$140 \mid$ This work is licensed under a Creative Commons Attribution 4.0 International License. 
huenda kutengeneza misuli kwa sababu ya kutaka kumfurahisha mwanamke.

Aidha, katika kipindi cha, chanzo cha kuvunjika kwa ndoa, Hadhira IV anaonyesha kuwa mwanamke anafaa kuhakikisha kuwa amejirembesha ili aweze kutambulika na kumfurahisha mwanaume. Anasema:

“...kama msichana uwe unajirembesha uonekane unakaa vizuri ili uweze kuattract yule ambaye unataka kumattract."

Kwa kusema hivyo yaani, kujirembesha ili uweze kumvutia unayetaka kumvutia, ana maanisha kuwa unafaa kujirembesha ili umvutie mwanaume. Hivyo, usemi huu unadhihirisha kuwa, lengo la kujirembesha kwa msichana au mwanamke ni kwa ajili ya mwanaume.

Kutokana na utafiti huu, mwanamke amechorwa kama chombo cha kumfurahisha mwanaume. Kwa mfano, katika kipindi cha uzuri wa nyumba ni mlango? Bi. Msafwari anasema, kuwa mwanamke hajitengenezi kwa sababu yake mwenyewe anajitengeneza kwa sababu ya mume wake na anaendelea kusisitiza kwamba, kujipamba kwa mwanamke si kwa sababu yake bali ni kwa sababu ya mume wake. Maneno yake ni ishara tosha kuwa mwanamke anachukuliwa kama chombo cha kumfurahisha mwaume. Mwanamke kuchukuliwa kama chombo cha kumfurahisha mwanaume, inadhihirisha, mihimili ya hadhi ya chini ya mwanamke katika jamii.

Matokeo haya yanaafikiana na yale ya Mrikaria (2017) katika kazi yake ya usawiri wa mwanamke katika nyimbo za kizazi kipya anaposema kuwa, mbali na kuwepo kwa wasanii wanaowathamini wanawake kuna wale wanaodunisha mwanamke yaani mwanamke anachorwa kama chombo cha kumstarehesha mwanaume.

\section{Ni msaidizi}

Mwanamke anapewa nafasi ya kuwa msaidizi wa mwanamume kwani asilimia themanini na tatu nukta tatu $(\mathrm{N}=20)$ ya vipindi vilivyoteuliwa vilikubaliana na hoja hii. Baadhi ya vipindi ambavyo vinavyoonyesha kuwa mwanamke anafaa kumuelekeze na kumsaidia mwanaume ni:
Kipindi cha kwa nini mwanaume haridhiki na mke mmoja. Mkala anasema:

“...mwanaume ndiye anayehitaji msaada mkubwa zaidi kuliko mwanamke katika mahusiano licha kuwa yeye ndiye kichwa katika familia, lakini katika kuangalia kushoto, kando, kulia, mbele, nyuma ni shingo ndio inasaidia."

Maneno haya yameweka wazi umuhimu wa mwanamke katika kuhakikisha kuwa mume wake anafanya mambo yanayofaa na kumfanya afanikiwe katika maisha. Kuna pale anataja shingo kumsaidia kichwa katika kufanya mambo, maneno hayo ni ya kijazanda tu kichwa hapo kinawakilisha mume na shingo kuwakilisha mwanamke. Kama tu vile kichwa hakiwezi kufanya chochote bila shingo yaani ni sharti shingo ielekeze kichwa katika maisha halisi vivyo hivyo mke anafaa kumfanyia hivyo mume wake.

Katika kipindi cha kwa nini wanawake wanaenda nje ya ndoa, jukumu la mwanamke kama mwelekezi linajitokeza tena kupitia kwa mazungumzo ya Mkala pale anaposema:

“... mwanaume anajua kutafuta lakini kudhibiti ni adhibitiwe. Mwanamume anapotafuta yule mwanamke, atafanya kila jambo awezalo lakini akishakupata analegeza kamba sana. Lazima kama wewe mwenyewe mwanamke ufikirie mbinu utakazozitumia kulifufua..."

Anaposema adhibiti ni adhibitiwe anamaanisha kuwa ni lazima mwanaume aelekezwe na mwenye kumwelekeza ni mke wake kwa sababu akishampata mke atajiona ashafika na kuna mambo ambayo hatayatekeleza kama hatakumbushwa kwa hivyo mwenye kumkumbusha ni mke wake na si mtu mwingine. Nacho katika kipindi cha, Maswali na mawaidha ya Bi. Msafwari ambapo Bi. Msafwari anasema:

“...mwanamke ni ubavu wa mwanaume hivyo, anapaswa mwanaume kubeba mzigo mkubwa kushinda mwanamke..."

Kauli hii inaonyesha kuwa mwanamke anafaa kuwa msaidizi tu wa mwanaume. Yeye hawezi kubebeshwa mzigo mzito kwa kuwa yeye ni ubavu kumaanisha mwili uliobaki ni mwanaume. 
Katika kipindi cha Mke kumtunza mume, Rashid anarejelea kauli ya mwanamke aliyekuwa anazungumza hivi:

"Once he takes off his bag, leterally go and wash him, scrab him, don't let him do anything himself. Does it clean his ears, clean like how you could wash a baby do that? Dry him, put mafuta on his body, dress him."

Kulingana na kauli hiyo, mwanamke anastahili kumsaidia katika baadhi ya majukumu kama vile, kumpa pesa za matumizi ya nyumba, kulipa karo na katika shughuli zinazohusishwa na mwanaume. Hata hivyo katika kipindi hiki Rashid anakiri kwamba, mwanaume ndiye anayebeba majukumu mazito katika familia hasa kifedha. Hivyo mwanadada huyu alikuwa anamaanisha kuwa sasa imefika wakati ambapo pia wanawake wajukumike na kuwasaidia waume zao kama namna ambavyo mama anamsaidia mtoto wake.

Katika kipindi cha, Je, pesa zake ni zake na zangu ni zetu? Bi. Msafwari anasema:

"Jambo la pesa ni ngumu sana na utakuta nyumba nyingi zinaharibika kwa sababu ya hizi pesa. Wanapofungishwa ndoa wanaambiwa kwamba wanakuwa mwili mmoja..."

Anaposema kuwa, wanakuwa mwili mmoja, anamaanisha kuwa wanafaa kusaidiana. Wanandoa wamelinganishwa na mwili wa mtu moja kwa vile wao wanafaa kusaidiana kwa kila jambo katika ndoa. Wao wamekuwa viungo katika mwili moja, na tunaelewa kuwa viungo vya mwili husaidiana kama vile; kama mwili unataka kufika mahali utategemea miguu. Hivyo jukumu la mwanamke ni kumsaidia mume wake kwa hali zote zile.

Kipindi cha zipi sifa za Husband material pia husisitiza katika umuhimu wa kusaidiana kwa mwanamke na mwanaume katika ndoa. Wakati Zulu anasema:

“ndoa ni kujengana kama unataka kwenda mbali kwenye ndoa yako..."

Kauli hii inasisitiza katika kusaidiana kwa wanandoa. Ambapo, mume na mke wanafaa kushirikiana ili kufanikisha ndoa yao. Kwenye kipindi cha dalili gani zakuonyesha mwanandoa yuko hatarini, Zulu anasema:

"sikuja katika ndoa kama mtoto wako nimekuja kama partner isipowork tuondokeane kwa heshima"

Zulu asisitiza kwamba kazi ya wanandoa ni kusaidiana na si kuadhibiana kwani mtoto ndiye anayeadhibiwa na si mtu mzima. Anaposema kuwa partner, kazi ya washiriki ni kusaidiana na wenzake ili biashara zao zifaulu. Hivyo pia katika ndoa mke na mume ni washiriki hivyo wanafaa kusaidiana katika ndoa. Kipindi cha nini maana ya usafi kwa wanandoa Bi. Msafwari anasema:

"mama ukiwa nyumbani uhakikishe kwamba bwana anapoingia zile soksi kama ashapokea azichukue azitie katika kikapu cha nguo chafu."

Kumaanisha kuwa mwanamke ana jukumu la kumuelekeza mume wake. Pale anapopokea soksi na kuweka katika kikapu cha nguo chafu, huyo mwanaume atajifunza kuwa, anapovua soksi zake anafaa kuchukua kwenye kikapu hicho.

Isitoshe katika kipindi cha usafi kwenye ndoa, $\mathrm{Bi}$. Msafwari anaeleza kuwa mama aliyefunzwa vizuri mume anapoingia anapaswa kuchukua soksi na aziweke kwenye ndoo ya nguo chafu, za ndani pia ziwekwe kwenye ndoo ya nguo chafu. Mwanamke ahakikishe kuwa kitanda ni nadhifu ili kimvutie mwanamume kuanzia siku aliyosema I do.

Kupitia kwa utafiti wetu mwanamke hasa mama ya watoto anasawiriwa kama msaidizi katika kipindi cha kulea watoto wa kambo wanaume wengi wanaulizwa kuwa na mke mmoja ambaye amekomaa kichwa na wakati wowote wanapotaka kutalikiana wafikirie juu ya watoto kwani ni mama tu anayeweza kulea mtoto na labda hana kipato anategemea baba.

Jambo hili la kumsaidia mwanamume lajitokeza katika kipindi cha vigezo vya wanaume kuhusu ndoa. Twaambiwa kuwa wataalamu wa mapenzi husema, alfajiri mchukue bwanako kama mwanao, mchana mchukue bwanako kama kakako na jioni mchukue bwanako kama hawarake. Hiyo ndiyo inayoitwa full time job ya kumsaidia mumeo mchana kutwa. 
Katika kipindi cha mwanamke mpumbavu na mwanamke mwenye hekima $\mathrm{Bi}$. Msafwari anasema kuwa mwanamke mwenye hekima huamka mapema na kumtengenezea mumewe kiamsha kinywa. Mwanamke huyu yuapatikana katika Proverbs 31 naye mume wake anamwita mke wangu wa heri. Anaongeza kuwa wanawake wa heri walibaki wachache. Isitoshe mwanamke atenge wakati wa kumfulia mume wake atafurahia.

Maishani kila mtu huwa na yule mtu ambaye anampa mwelekeo kwa kumpa mawaidha yatakayomsaidia katika kufanikisha maisha yake. Bila kuwepo kwa mwelekezi mambo huenda yakawa magumu. Hivyo ili nyumba iweze kusimama ni sharti mume amwelekeze mke naye mke amwelekeze mume.

\section{Kudumisha Amani katika Ndoa}

Asilimia sabini na tano $(\mathrm{N}=18)$ ya vipindi vilivyoteuliwa vilimpa mwanamke jukumu la kudumisha amani katika ndoa yake, na mara nyingi katika vipindi hivi utamkuta Bi. Msafwari akiwashauri wanawake kwa kuwaambia kuwa, mwanamke mpumbavu hubomoa nyumba yake na mikono yake mwenyewe na mwenye hekima hujenga nyumba yake, baadhi ya vipindi ambavyo mwanamke anapewa jukumu la kudumisha ndoa ni kama, uzuri wa nyumba ni mlango? ambapo anwani yenyewe inaonyesha kuwa mwanamke ndiye anafaa kuhakikisha kuwa ndoa yake inadumu. Mlango hapo ni wa kijazanda ambapo unawakilisha mwanamke nayo nyumba ni ndoa. Anwani hii inadhihirisha kuwa ili ndoa iweze kudumu, mwanamke anafaa kushikilia na kuhakikisha kuwa anafanya kila juhudi kuhakikisha ndoa inadumu.

Kipindi cha kwa nini wanawake wanaenda nje ya ndoa pia kinaonyesha kuwa mke anafaa kudumisha ndoa ambapo mkala anasema:

"zamani wanawake walikuwa wanashauriwa kwamba ukae ukijua mama, mwanaume atakwenda nje lakini tulia atakwenda kote kote lakini mwisho atarudi hapa."

Usemi huu unamaanisha kuwa mwanamke anafaa kuvumilia mateso yote anayoyapitia ili aweze kudumisha ndoa yake. Kwa hivyo kupitia kwa uvumilivu wa mwanamke ndio huzaa matunda ambayo ni kudumu kwa ndoa.
Kipindi kingine kinachoshughulikia mada hii ni cha Majukumu ya mwanamke, ambapo Bi. Msafwari anasema:

“...kila wakati mi nawaambia kwamba mwanamke mpumbavu hubomoa nyumba yake na mikono yake mwenyewe na mwenye hekima akaijenga nyumba yake. Nataka ujue majukumu yako kama ni kumtengenezea bwanako breakfast, lunch, umpelekee maji ya kuoga hata kama una mfanyikazi..."

Maneno haya yanaonyesha kuwa mke anafaa kutekeleza majukumu yake yote na anapotekeleza haya majukumu bila shaka amani itaweza kuwepo katika nyumba yake.

Kwenye kipindi cha kwa nini mwanaume haridhiki na mke moja, Mkala anasema:

"ukishateta mwanamke na kununa pale nyumbani hakikisha unaleta kirutubishi kitamu ili mambo yakawa yale mazuri halafu ndio mnalala."

Mkala ametaja kuhusu kirutubishi, ambapo katika hali halisi kirutubishi ni kile kitu kinachokifanya kitu kuwa na afya, hivyo anadai kuwa mke ndiye anayefaa kuleta kirutubishi katika nyumba. Kirutubishi hicho huenda kikawa upendo, vyakula na furaha katika nyumba na mambo haya yanajenga amani ambapo amani hudumisha ndoa. Mwanamke hivyo amepewa jukumu hilo la kuleta kirutubishi katika ndoa, kama vile tu wanyama na wanadamu wanahitaji virutubishi mbalimbali ili waweze kukua na kuwa na afya vilevile kama vile mimea huitaji virutubishi ili yakaweza kunawiri vizuri na kuwa na afya ndivyo ndoa huitaji amani ili iweze kudumu.

Kipindi kingine ambacho kimempa mwanamke jukumu la kudumisha amani ni kipindi cha kwa nini wanawake wanaenda nje ya ndoa ambapo Bi. Msafwari anasema:

"hawa wanawake mnaotusikiza waelewe kwamba, kuna mabwana wengine wanatoka kwa ajili wao ni mavuvuzela. Ukitulia, bwana akuje akute umetulia atakusikia..."

Hivyo, kulingana naye amani huchangia sana katika kudumu kwa ndoa. Mwanamke anashauriwa awe na utulivu katika nyumba asiwe wa kusema sana hadi 
kumkosesha mumewe amani kwa vile haya yatachangia katika kutoka nje ya ndoa kwa mzee na hayo yakishatendeka ndoa hiyo itakuwa inayumbayumba na wakati wowote inaweza ikavunjika. Vuvuzela ni chombo cha muziki ambacho hupulizwa na huwa na sauti kubwa sana hivyo, mwanamke anaambiwa asiwe vuvuzela, ambapo haimaanishi kuwa mwanamke ni hicho chombo cha kupuliza bali inamanisha kuwa asiwe mtu wa kusema maneno mengi na ambayo hayafurahishi, isiwe kila mara ni kukelelesha mumeo kwa vile itachangia katika kuvunjika kwa ndoa.

Aidha kipindi cha, Athari za pesa kwenye ndoa, Mkala anasema:

"sasa paka mkunekune kichwa atasikia vizuri... mkunekune paka wako usipomkuna kuna mtu atakayemkuna."

Mkala anamaanisha kuwa kama mwanamke unafaa kuhakikisha kuwa mume wako anaridhika nawe ili asianze kutafuta wengine pale nje kwa kuwa usipomridhisha atawatafuta wanawake wengine hivyo, kuanguka kwa ndoa. Katika kipindi cha, Mke kumtunza mume, Rashid anasema:

\section{"viungo ni kuoga, kuvishwa ndio utamani} mboga"

Kutokana na kauli hii mboga ni mwanaume ambapo anadaiwa katika kipindi kuwa anafaa kusaidiwa kwa kuoshwa na kuvishwa. Kuoshwa na kuvishwa ni kumsaidia katika majukumu yake kama kulipa nyumba, karo na hata bili ya stima hivyo ndivyo viungo. Kipindi cha, Je pesa zake ni zake na zangu ni zetu? Zulu anasema:

“... kazi ya mwanamke ni kumaintain Boma, yaani mume na watoto."

Anaposema kumaintain boma yaani kudumisha boma anamaanisha kuwa mwanamke anafaa kuhakikisha kuwa ndoa yake inadumu kwani ndoa ni ya mke na mume na mazao ya ndoa ni watoto. Vilevile udumishaji wa ndoa kwa mwanamke unasisitizwa katika kipindi cha, je, kumshuku mpenzi wako ndio ishara ya kumpenda? ambapo mwanamke hafai kujishughulisha katika kumfuata na kumshuku mume ili ndoa yake idumu. Mfano kutoka kauli ya Zulu ambapo anasema:
"Lakini hata nikutie imani sijakutia sababu ya kunichunguza. Nini waniweka na tochi kila mahali?"

Kutokana na kauli ya Zulu ni dhahiri kuwa mke hana haki ya kumchunguza mume. Anasema mbona waniweka tochi yaani kurunzi tunafahamu kuwa kurunzi hutumika katika kumulika sehemu ambayo haina mwanga. Hapa kurunzi imetumika kueleza kuwa mwanaume hafai kumulikwa mulikwa kama anafanya mambo yasiyofaa asifuatwe.

Katika kipindi cha nini huchangia usaliti au mipango ya kando kwenye ndoa wanawake wanapaswa kunyamazia matatizo yanayowakumba ili kudumisha amani katika ndoa. Zulu anasema kuwa wanawake wanavumilia mambo mengi. Kuna wanaume tunaoona barabarani na generator zao haziwaki, ziliacha kukimbia, kama tuktuk zinawashwa na kamba inasukumwa ndiyo iweze kuguruma kidogo lakini hawatasema na nikasema kuwa tunapokula nyama tuchunge na vitabi na pombe tuchunge sana, tuchunge afya. Kuna wanaume wanaoanguka kwa kitanda paa! Na sasa mwanake avumilie pale. Hakubaliwi kuita wazee na kusema huku kwangu hakuwaki, wanaume tusijione kama wenye nguvu.

Pia kwenye kipindi cha dalili gani zakuonyesha mwana ndoa yuko hatarini, Bi. Msafwari anasema mwanamke aweze kutumia sauti zake kuomba msamaha, mwanamke awe mnyenyekevu kwa mumewe ili kudumisha amani. Isitoshe kwenye kipindi cha mwanamke mpumbavu au mwanamke mwenye hekima, Bi. Msafwari anashauri mwanamke asiwe mwepesi wa kufanya uamuzi haraka kwani hata mume anaweza kutumiwa ujumbe wa uongo na mwanamke mwingine ili kuharibu uhusiano kwenye ndoa.

\section{Kutii, Kumheshimu na Kunyenyekea Mbele ya Mume}

Vipindi asilimia hamsini ( $\mathrm{N}=12)$ katika vipindi vilivyoteuliwa vinaonyesha kuwa jukumu la mwanamke ni kutii, kumheshimu na kunyenyekea mbele ya mume. Mfano ni kipindi cha, kwa nini mwanamke anakwenda nje ya ndoa? Bi. Msafwari anasema: 
"Hawa wanawake wanaotusikiliza wajue kwamba, mabwana wanatoka kwa ajili wao ni mavuvuzela."

Anaposema wanaume wanatoka kwa sababu wanawake ni mavuvuzela anamaanisha wanawake hao hawana heshima. Vuvuzela ni ala ya muziki ambayo hutoa sauti na sauti hiyo husumbua wakati mwingine. Hivyo mke anaposema sana mwanaume anamchoka na kuona kuwa amekosewa heshima. Kipindi kingine kinachoweka wazi suala hili ni kipindi cha, kwa nini mwanaume haridhiki na mke mmoja ambapo Mkala anasema:

"Mwanaume ana kujivunia kwake, ana ufalme wake ambao anauhisi kwamba yuko nao. Kiasi cha kwamba we ukimshambulia usiondoe huo."

Simba ni mnyama wa porini ambaye huaminika kuwa mwenye nguvu kushinda wanyama wote porini na mara nyingi hurejelewa kama mfalme wa porini hivyo yeye ndiye anaongoza wanyama. Kazi ya wanyama wengine ni kumtii na kunyenyekea mbele ya mfalme wao na heshima pia laziwa iwepo. Maneno ya Mkala, yanaonyesha wazi kuwa mke anafaa kumheshimu mumewake, anafaa kumtii na hata kunyenyekea mbele ya mume wake. Anasema kuwa, hata mkizozana kivipi, hakikisha kuwa usiondoe ufalme wake yaani hakikisha kuwa hujamshusha hadhi kwa kuwa yeye ndiye kiongozi wa nyumbani na atabaki kuwa kiongozi hivo, mke anafaa kumheshimu. Ni dhahiri kuwa mwanaume amepewa sifa ya kutawala nyumbani kama vile simba hutawala porini hivyo kudaiwa kuwa mke achunge asipite mipaka yake kwa vile kuna mpaka wake na ule wa mume wake.

Katika kipindi cha Majukumu ya mwanamke, Hadhira VII anataja heshima na utiifu kwa mume kutoka kwa mke wake. Anasema:

"Ni vyema sana kutii na kuheshimiana. Katika kitabu cha Waepheso 5, kinaonyesha kwamba, mke anapaswa kujitiisha kwa mume wake na mume anapaswa kumpenda mke wake..."

Ni dhahiri kuwa jukumu la mke ni kumtii naye mume anapewa jukumu la kumpenda mke wake. Pia katika kipindi cha Maswali na mawaidha ya Bi. Msafwari, ambapo Bi. Msafwari anasema, “...na kutaka kujua si ukali ni kumbembeleza. Mwanaume ni kama mtoto, yaani vile utakavyomlea mtoto na yeye anataka yale malezi."

Anasema kutaka kujua si ukali kumaanisha ni kumuuliza kwa heshima ili uweze kupewa majibu na mume. Anafananishwa na mtoto kwa vile mtoto unapomrekebisha kwa upendo akuwa na uwazi nawe wakati anapokua vivyo ndivyo alivyo mwanaume. Anataka umuulize kwa upendo, heshima na unyenyekevu ili aweze kuwa na imani na nawe. Aidha, katika kipindi cha Mke kumtunza mume, Hadhira VII anasema:

"Mume anafaa kuwa kichwa katika familia. Kama kichwa, wafaa kuanza wewe, kumanisha kama ni kichwa yule ni msaidizi."

Mume amerejelewa kama kichwa yaani kiongozi katika familia na mke ni msaidizi wake tu. Kama mume ni kiongozi basi ni sharti aheshimiwe na mke pamoja na watoto. Mume kama kiongozi yeyote yule ni hadi aheshimiwe. Kwani hata viongozi wa kisiasa wanapozuru mahali hupewa heshima nyingi hivyo, pia mume anastahili heshima pamoja na utiifu kutoka familia yake.

Katika kipindi cha Je, pesa zake ni zake na zangu ni zetu? Bi. Mariam anadokeza kwamba:

“...mume karejea nyumbani, ni lazima mwanamke ucheze na nyama yako ya ulimi..."

Kauli hii inamanisha kuwa, kama mke, unafaa kunyenyekea mbele ya mume wako. Anasema, cheza na ulimi wako. Kumaanisha usiyatamke maneno mabaya. Sema mambo mazuri kwa mume wako. Kama mke, mtu anafaa kuhakikisha kuwa humkasirishi mume wake kwa kuyatamka maneno mabaya yasiyopendeza. Kipindi cha Je, kumshuku mpenzi wako ndiyo ishara ya kumpenda? Zulu anasema:

"Lakini hata nikutie imani, sijakutia sababu ya kunichunguza. Nini waniweka na tochi kila mahali?"

Kutokana na maneno hayo tunatambua kuwa wanaume wanataka kupewa nafasi yao wasikuwe wanafuatwa fuatwa na kuchunguzwa na wake wao. Hivyo, kuonyesha kuwa wanataka heshima kwa vile 
wanapochunguzwa wanaona kama wamekosewa heshima. Waaidha, katika kipindi cha Dalili gani zakuonyesha mwanandoa yuko hatarini, Bi. Msafwari anaeleza madhara ya ukosefu wa heshima kwa kusema

“... waeza kuwa watu wanagombana ndani ya nyumba na utasikia mwanamke akisema nichape basi... unafaa kunyenyekea na ujue kwamba wewe ni mwanamke..."

Kwa kweli maneno hayo ambayo mwanamke anaweza kutamka kama nichape basi ni ukosefu wa heshima na utajikuta kama mwanamke ukichapwa na mume kwa sababu ya maneno unayoyatamka. Anaendelea kukusisitiza katika unyenyekevu wa mwanamke kwa mwanaume anasema kuwa tunafaa kunyenyekea kwa sababu wewe ni mke.

Katika kipindi cha chanzo cha kuvunjika kwa ndoa za sasa, mwanamke anapewa nafasi ya kunyenyekea na kumheshimu mume wake. Hayo yanadhihirika kupitia kwa arafa anayosoma Kanze inayosema:

“...wanawake wamepewa kipau mbele ya kuzungumza na hawachunguzi maneno yao wala hawaogopi na kuwaheshimu mabwana zao katika familia."

Kulingana na mtazamaji huyo mwanamke anastahili kunyenyekea mbele ya mume vilevile anafaa kumheshimu mume wake. Anaonelea kuwa kutokuwa na heshima na unyenyekevu kutoka kwa mke ndiyo imechangia katika kuvunjika kwa ndoa za sasa.

Matokeo ya utafiti huu ni kuwa jukumu la mwanamke ni kumtii, kumheshimu na kunyenyekea kwa mwanaume. Matokeo hayo yanalingana na ya Saro (2017) anayesema, mwanamke ni kiumbe anayetazamiwa kuwa mtiifu kwa wazazi, walimu na kwa waume zao. Ufeministi wa Kiafrika umeshughulikiwa katika sehemu hii ambapo, Mihimili inayoona mwanamke kama ana hadhi ya chini inajitokeza. Kwani, nadharia hii inazingatia utamaduni unaomkandamiza mwanamke kwani yeye ndiye wa kutii na kunyenyekea na sio mwanaume.

\section{HITIMISHO}

Utafiti huu umethibitisha kuwa, kila mwanajamii ana jukumu lake katika jamii, kila mtu ana nafasi yake katika jamii. Hivyo nafasi ya mwanamke ni kama ifuatavyo kwa mujibu wa kipindi cha Bi. Msafwari: mwanamke ana nafasi ya kuwa chombo cha kumfurahisha mwanamume, ana nafasi ya kuwa msaidizi wa mwanamume, nafasi ya kudumisha amani kwenye ndoa na kutii mume wake.

\section{MAPENDEKEZO}

Utafiti huu umechunguza taswira ya mwanamke kwenye runinga, hivyo, tunapendekeza utafiti zaidi ufanywe kuhusu mwanamke kwenye vyombo vingine vya habari kama vile vipindi vya redio na hata vipindi vingine vya televisheni. Aidha, utafiti huu umeshughulikia mwanamke pekee na tafiti nyingi ambazo tulizisoma zilishughulikia mwanamke. Kwa hivyo, tunapendekeza utafiti zaidi ufanywe kuhusu usawiri wa mwanaume katika vyombo vya habari.

\section{MAREJELEO}

Adeola, J. (1990). In Theire Own Voices. African Women Writers Talk London. James Curren Limited.

Africa Gender Equality Index, (2015). Empowering African Women: An Agenda for Action- African Development Bank Group: African Development Bank.

Bi. Msafwari. ( 3/9/2016). Mawaidha na Bi. Msafwari: Uzuri wa Nyumba ni Mlango? Runinga ya Citizen, Kenya.

Bi. Msafwari. (1/10/2016). Mawaidha na Bi. Msafwari: Majukumu ya Mwanamke.Runinga ya Citizen, Kenya.

Bi. Msafwari. (1/5/2021). Mawaidha na Bi. Msafwari: Dalili gani zakuonyesha mwanandoa yuko hatarini? Runinga ya Citizen, Kenya.

Bi. Msafwari. (1/9/2018). Mawaidha na Bi. Msafwari: Mke kumtunza Mume. Runinga ya Citizen, Kenya. 
Bi. Msafwari. (10/2/2018). Mawaidha na Bi. Msafwari: Maswali na Mawaidha ya Bi. Msafwari. Runinga ya Citizen, Kenya.

Bi. Msafwari. (12/6/2021). Mawaidha na Bi. Msafwari: Je, pesa zake ni zake na zangu ni zetu? Runinga ya Citizen, Kenya.

Bi. Msafwari. (13/1/2018). Mawaidha na Bi. Msafwari: Vigezo vya wanaume kuhusu ndoa. Runinga ya Citizen, Kenya.

Bi. Msafwari. (14/11/2020). Mawaidha na Bi. Msafwari: Ni nini huchangia usaliti na mipango ya kando? Runinga ya Citizen. Kenya.

Bi. Msafwari. (14/4/2018). Mawaidha na Bi. Msafwari. Mjakazi kumkosoa mke mwenye nyumba. Runinga ya Citizen, Kenya.

Bi. Msafwari. (17/9/2016). Mawaidha na Bi. Msafwari: Kwa nini Wanawake Wanaenda Nje ya Ndoa. Runinga ya Citizen, Kenya.

Bi. Msafwari. (18/2/2017). Mawaidha na Bi. Msafwari: Kwa nini mwanaume haridhiki na Mke Mmoja. Runinga ya Citizen, Kenya.

Bi. Msafwari. (21/11/2020). Mawaidha na Bi. Msafwari: Nini maana ya usafi kwa wanandoa. Runinga ya Citizen, Kenya.

Bi. Msafwari. (22/5/2021). Mawaidha na bi. Msafwari: Kulea watoto wa kambo. Runinga ya Citizen, Kenya.

Bi. Msafwari. (22/6/2019). Mawaidha na Bi. Msafwari: Maswali kuhusu Masuala ya ndoa. Runinga ya Citizen, Kenya.

Bi. Msafwari. (23/3/2019). Mawaidha na Bi. Msafwari: Mbinu za kuboresha ndoa. Runinga ya Citizen, Kenya.

Bi. Msafwari. (25/3/2017/). Mawaidha na Bi. Msafwari: Chanzo cha Kuvunjika kwa ndoa za sasa. Runinga ya Citizen, Kenya.

Bi. Msafwari. (26/5/2018). Mawaidha na Bi. Msafwari: Mwanamke mwenye hekima na mpumbavu ni yupi? Runinga ya Citizen, Kenya.
Bi. Msafwari. (26/6/2021). Mawaidha na Bi. Msafwari: Katika ndoa ni vipi vya kusemwa na vipi vya kuwekwa chini ya maji. Runinga ya Citizen, Kenya.

Bi. Msafwari. (29/4/2017). Mawaidha na Bi. Msafwari: Athari za pesa kwenye ndoa.Runinga ya Citizen, Kenya.

Bi. Msafwari. (3/6/2021). Mawaidha na Bi. Msafwari: Je, kumshukushuku mpenzi wako ndiyo ishara ya kumpenda? Runinga ya Citizen, Kenya.

Bi. Msafwari. (31/10/2020). Mawaidha na bi. Msafwari: Zipi sifa za Husband material? Runinga ya Citizen, Kenya.

Bi. Msafwari. (4/8/2018). Mawaidha na Bi. Msafwari: Kisa cha kuachwa na mume. Runinga ya Citizen, Kenya.

Bi. Msafwari. (5/6/2021). Mawaidha na Bi. Msafwari: Ni kosa kujuana na mtu mtandaoni ilhali soko zote zimeingia mtandaoni? Runinga ya Citizen, Kenya.

Bi. Msafwari. (9/3/2020). Mawaidha na Bi. Msafwari: Katika ndoa, ni vitu gani huchukiza wanaume? Runinga ya Citizen, Kenya.

Buffon, G. (1930). Buffon's Discourse on Style. Paris Librairie: Hatier publishers.

Creswell, J. (2009). Mapping the field of mixed methods research.

European Union, (2021). 2021 report on gender equality in Europe. Imetolewa kwa https://ec.europa.eu

Irib World Service. (2014). Matatizo ya Mwanamke katika ulimwengu wa Magharibi. Imetolewa kwa www.kiswahili.irib.irib.ir/38823.

Kasuma, R. (2014). Uchanganuzi wa uhusiano wa kijinsia katika vipengele vya tamthilia za Kilio cha haki na Sudana. Tasnifu ya uzamili. Chuo kikuu cha Egerton.

Kawia, M. (2015). Kuchunguza Usawiri Chanya wa Mwanamke Katika Vitendawili vya Jamii ya

147 | This work is licensed under a Creative Commons Attribution 4.0 International License. 
Wanyiha (Doctoral dissertation, The open University of Tanzania).

Kombo, D. K. Na Tromp, D. L. (2006). Poposal and Thesis Writing: An Introduction.Nairobi: Paulines Publications Africa.

Kothari, C. (2004). Research Methodology, Methods and Techniques. Second Edition. New Delhi: Wiley Eastern Limited

Lantara, N. F. (2015). The roles of woman as leader and housewife. Journal of Defense Management, $5(01)$.

Leech, G. (1969). A Linguistic Guide to Mordern English Poetry. Essex: Longman Group Ltd.

Mavisi, R. (2007). Usawiri wa wahusika wa kike katika kazi za zainabu Burhani. Doctoral dissertation.

Mrikaria, S. (2011). Usawiri wa Mwanamke katika Nyimbo za Kizazi Kipya .Kioo cha Lugha. 9 (1), 122-143.

Mugenda, O. M. \& Mugenda, A. G. (2003). Research Method, qualitative and quantitative approaches. Nairobi: Acts press.

Murungi, G. (2013). Mtindo unavyoendeleza Maudhui katika Natala. Tasnifu ya Uzamili Chuo kikuu cha Nairobi.

Muthuria, M. (2009). Maadili ya Utafiti. Chuo kikuu cha Masaai Mara

Njogu, K. na Wafula, R. (2007). Ufundishaji wa fasihi. Nairobi.: Jomo Kenyatta Foundation.

Ntaragwi, M. (2004). Uhakiki wa kazi ya Fasihi. Rock Island: Augustana College of Wisconsin Press, Madison, WI.

Oiko, F. (2017). Uhakiki wa mtindo katika Tamthilia za Timothy arege. Tasnifu ya uzamili chuo kikuu cha Maasai Mara.

Ramton, M. (2015). Four waves of Feminism. Percific University. Imetolewa kwa http://www.pacificu.edu/about-us/news-events.

Saro, S. (2017). Kuchunguza Usawri wa mwanamke katika Riwaya: mfano wa Riwaya ya
Utengano. Tasnifu ya Uzamili. Chuo Kikuu Huria cha Tanzania.

Sawe, A. (2018). Usawiri wa mwanamke katika ndoa: mifano kutoka utendi wa mwanakupona, utendi wa Ayubu na Matini Teule za kidini. Tasnifu ya uzamifu. Chuo Kikuu cha Moi.

Sawe, A. Makoti, V. na Obuchi, S. (2016). Taswira ya mwanamke katika ndoa mintarafu utendi wa mwanakupona. Mara Research journal of Kiswahili: The African Premier Research Publishing Hub.

Simpson, P. (2004). Stylistics: A Resource Book for Students. London Routledge.

Steady, F. (1981). The Black African Cross Cultuary. Cambridge: Schenkman Publishing Company.

Wamitila, K. W. (2003). Kamusi ya Fasihi: Istilahi na Nadharia: Nairobi Focus Publishers.

Wanjala,F. S. (2020). Safina ya Utafiti wa Fasihi Simulizi. Elgon Epitome Publishers Ltd.

148 | This work is licensed under a Creative Commons Attribution 4.0 International License. 